\section{Rütteln am Dogma der radikalen Dissektion}

Das Konzept der Biopsie des Sentinel-Lymphknoten ist etablierte Strategie für ein präzises Staging des malignen Melanoms. Allerdings besteht kein Konsens hinsichtlich der praktischen Konsequenzen eines positiven Befundes - der Überlebensvorteil einer kompletten Lymphknotenausräumung bei positivem Lymphknotenbefall ist bis jetzt nicht bewiesen.

$\mathrm{D}$ as Münchner Autorenteam prüfte, ob es eine Möglichkeit gibt, Patienten mit Lymphknotenmetastasen zu identifizieren, bei denen auf die regionäre Dissektion verzichtet werden kann. Dazu werteten sie die Daten von 213 Patienten mit Befall des bzw. der Sentinel-Lymphknoten (SNL) und der Empfehlung zur kompletten Dissektion aus.

Nur bei 176 der Patienten (82,6\%) wurde die radikale Ausräumung tatsächlich ausgeführt. In diesem Kollektiv wiesen 26 Patienten (14,8\%) einen Tumorzell-Befall in weiteren Lymphknoten (Nicht-SentinelLymphknoten = NSLN) auf. Die 5-JahresÜberlebensrate betrug in dieser Subgruppe $26,1 \%$, gegenüber $74 \%$ bei den NSLNnegativen Patienten. Die mediane Tumor- dicke war signifikant mit einem positiven Nachweis einer metastatischen Beteiligung weiterer Lymphknoten korreliert: 3,03 mm bei Befall von NSLN versus 2,22 $\mathrm{mm}$ bei NSLN-negativen Patienten.

Patienten mit positivem SentinelLymphknoten, die jedoch keiner radikalen Dissektion unterzogen wurden, hatten eine mittlere krankheitsfreien Zeit von 97 Monaten (5-Jahres-Rate: 71\%) und überlebten im Mittel 107 Monate (5-Jahres-GesamtÜberleben: 75,9\%). Damit war das Outcome in diesem Kollektiv, gemessen an den beiden Endpunkten, besser als in der Dissektions-Gruppe.

Patienten, die die also trotz Befall des Wächter-Lymphknotens die ausgedehnte Ausräumung verweigerten, hatten eine bessere Prognose als jene, die sich der Prozedur unterzogen. Limitiert ist das Resultat dieser Studie durch die kleine Fallzahl: Die Gruppe, bei der keine komplette regionäre Lymphadenektomie durchgeführt wurde ( $\mathrm{n}=37$ ), war möglicherweise zu klein, um eventuell vorliegende Tumoroder Patientencharakteristika zu entdecken, die den günstigen Verlauf erklären.

Fazit: Wenn der Tumor bereits in weitere Lymphknoten gestreut hat, haben SLNpositive Patienten eine hochsignifikant schlechtere Prognose als wenn nur der Sentinel-Lymphknoten betroffen ist. Da die Tumordicke prädiktiv für den Nodalstatus der Lymphregion ist, kommt sie möglicherweise zukünftig als ein Marker in Frage, den man bei der Entscheidung für oder gegen eine komplette Dissektion hinzuziehen könnte. Waltraud Paukstadt

Kunte $C$ et al. Analysis of predictive factors for the outcome of complete lymph node dissection in melanoma patients with metastatic sentinel lymph nodes.

J Am Acad Dermatol 2011; 64: 655-62.

\title{
Striktes Hygienemanagement beendet MRSA-Ausbruch
}

Bei elf stationären Hautpatienten der Universitätsklinik Zürich wurde vor mehreren Jahren eine Infektion mit einem Methicillin-resistenten Staphylococcus-aureus (MRSA)-Stamm nachgewiesen. Die Klinik reagierte mit einem strikten und aufwendigen Hygienekonzept.

$\mathrm{D}$ ie registrierten MRSA-Fälle von 2003 konnten in der genotypischen Analyse alle einem speziellen Stamm zugeordnet werden, der zuvor an der Klinik nicht registriert worden war. Gemäß einem internen Kennzeichnungs-System erhielt er die Nummer 35. Der Ausbruch veranlasste zur Implementierung strikterer Hygienemaßnahmen, darunter die Installation von Spendern zur Händedesinfektion im Abstand von zehn Metern in den Gängen sowie am Fußende jeden Krankenbetts. Vorgeschrieben wurde die Verwendung von Einmalhandschuhen grundsätzlich bei jeder Prozedur mit Kontakt zur befallenen Haut oder Wunden von Patienten. Ferner dürfen ExternaTöpfe oder -Tuben nicht mehr zum Bett der Patienten mitgenommen werden; die für die Lokaltherapie benötigte Menge wird in der Stationsapotheke hergerichtet.

Bei 65 Patienten der dermatologischen Abteilung wurde zwischen 2003 und 2008 eine MRSA-Kontamination diagnostiziert. In der Mehrzahl der Fälle stammte der erste MRSA-positive Abstrich von einer offenen Wunde. $45 \%$ waren mit dem Keim kolonisiert, $55 \%$ infiziert. Von diesen hatten vier eine Bakteriämie, zwei erlagen einer MRSA-Sepsis.

Die Inzidenz ,MRSA-35'-positiver Fälle nahm im Beobachtungszeitraum signifikant ab; 2008 wurden keine neuen Fälle mehr registriert. Während die MRSA-35Übertragung gestoppt wurde, ging die Zahl der MRSA-Kontaminationen mit anderen Stämmen stark nach oben (15 ambulante Patienten im Jahr 2008). Diese Zunahme anderer MRSA-Typen stellt den Autoren zufolge die erfolgreichen Hygienemaßnahmen keineswegs in Frage. Vielmehr belegt die Tatsache, dass die Elimination eines bestimmten MRSATyps in einer Zeit erreicht wurde, in der neue MRSA-Fälle gehäuft auftraten, die Effektivität der Strategie.

Eine vollständig abgeheilte Hautbarriere erwies sich als entscheidende Voraussetzung einer erfolgreichen Sanierung.

Fazit: Konsequente Umsetzung des Hygieneregimes kann demnach einen spezifischen Stamm in einer Klinik komplett eradizieren. Die Untersuchung dokumentiert, dass umfassende Hygieneinterventionen einen Ausbruch multiresistenter Bakterien terminieren können.

Waltraud Paukstadt

Gilomen S et al. Successful control of methicillin-resistant Staphylococcus aureus outbreak at a university department of dermatology. J Eur Acad Dermatol Venereol 2011; 25: 441-6 